\title{
Research Paper: The Relationship Between Muscle Strength and Range of Motion in Lower Extremity With Balance and Risk of Falling in Elderly
}

\author{
Hamed Zarei ${ }^{*}$, Ali Asghar Norasteh ${ }^{1} \odot$ Masoomeh Koohboomi $^{1}$
}

1. Department of Corrective Exercise and Sport Injuries, Faculty of Physical Education and Sports Sciences, University of Guilan, Guilan, Iran.

\begin{tabular}{|c|c|}
\hline $\begin{array}{l}\text { Use vour device to scan } \\
\text { and read the article online }\end{array}$ & Ctetion Zarei H, Norasteh AA, Koohboomi M. The Relationship Between Muscle Strength and Range of Motion in Lower \\
\hline 口ifipra & $\begin{array}{l}\text { Extremity With Balance and Risk of Falling in Elderly. Physical Treatments. 2020; 10(1):33-40. http://dx.doi.org/10.32598/ } \\
\text { ptj.10.1.430.1 }\end{array}$ \\
\hline 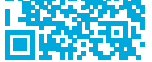 & d ef" http://dx.doi.org/10.32598/ptj.10.1.430.1 \\
\hline
\end{tabular}

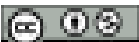

Article info:

Received: 16 Oct 2019

Accepted: 10 Dec 2019

Available Online: 01 Jan 2020

Keywords:

Elderly, Balance, Falling risk, Muscle strength, Range of motion

\section{A B S T RA C T}

Purpose: The present study examined the relationship between muscle strength and range of motion in lower extremity with balance and risk of falling in the elderly.

Methods: This was a cross-sectional study. The statistical population included healthy elderly (Mean \pm SD age: $65.64 \pm 4.95 \mathrm{y}$ ) in Rasht City, Iran. The study was conducted on 65 healthy elderly males who met the study inclusion criteria and were selected by convenience sampling method. In this study, to measure static and dynamic balance, Sharpened Romberg test and Get Up and Go test were conducted, respectively. Additionally, the falling risk was measured by the Berg Balance Scale. Moreover, the maximum isometric force of the muscle groups in the lower extremities was measured using a hand-held dynamometer, and the range of motion was measured by a goniometer. The obtained data were analyzed using descriptive and analytical statistics by SPSS.

Results: The present study results demonstrated that the muscle strength of hip extension, hip abduction, hamstring, quadriceps, and ankle plantarflexion had a significant relationship with static balance; while being negatively related to dynamic balance $(\mathrm{P} \leq 0.05)$. Furthermore, we observed a significant and negative relationship between the muscle strength of ankle dorsiflexion and dynamic balance and risk of falling $(\mathrm{P} \leq 0.05)$. There was also a positive and significant relationship between the range of motion hip extension, knee flexion, plantar flexion, and ankle dorsiflexion and static balance, while indicating a negative and significant relationship between these variables and dynamic balance and risk of falling $(\mathrm{P} \leq 0.05)$.

Conclusion: The muscle strength hip abduction, hip extensions, hamstring, quadriceps, plantar flexion, ankle dorsiflexion and range of motion hip extension, knee flexion, ankle plantar flexion, and dorsiflexion seem to affect balance maintenance and fall risk in the elderly.

\footnotetext{
* Corresponding Author:

Hamed Zarei, PhD.

Address: Department of Corrective Exercise and Sport Injuries, Faculty of Physical Education and Sports Sciences, University of Guilan, Guilan, Iran. Phone: +98 (914) 2504802

E-mail: zareei.h@yahoo.com
} 


\section{Highlights}

- The results showed a high positive correlation between muscle strength in lower extremity with balance in Elderly

- The results showed a high negetive correlation between muscle strength in lower extremity with risk of falling in Elderly.

- The results showed a high positive correlation between range of motion in lower extremity with balance in Elderly.

- The results showed a high negative correlation between range of motion in lower extremity with risk of falling in Elderly.

\section{Plain Language Summary}

Our results suggested that muscle strength and the range of motion in the lower extremity significantly affect balance and decrease the risk of falling in the elderly. Thus, researchers could develop intervening and training exercises to promote balance and reduce the risk of falling in the elderly to improve their life quality, biopsychological well-being, and encourage an active lifestyle among them.

\section{Introduction}

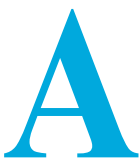

ging has turned to a serious challenge in developing and developed countries and poses numerous psychosocial, cultural, economic, and ethical considerations. The World Health Organization (WHO) reported that the number of people aged $>60$ years would reach 2.1 billion in 2025, with most of them in developing countries, like Iran [1]. Providing healthcare services to this increasing population is of dire importance. This is because aging is strongly related to decreased health and adverse transformations in body tissues and organs, as well as psychological changes, which require greater caregiving [2].

Attempts to prevent and diagnose age-related problems and improve the life quality of the elderly are significantly important. Falling is a major threat to them because it affects their health in numerous manners [3]. It causes physical (pelvic fracture, functional disability, \& death) and psychological (the loss of self-confidence, low self-esteem, \& decreased life-expectancy) complications [4]. Therefore, determining the causes of falling and developing diagnostic procedures to treat the affected population provides an opportunity to improve their life-quality, independence, and significantly decrease costs of care [5]. Multiple factors, such as hearing and visual impairments, are identified as the major causes of falling. However, the imbalance is found to be a key determinant in this regard, as well [6].
Balance is a complex motion skill that describes postural dynamics in preventing falling; the appropriate performance of daily activities, like walking and taking stairs, depends on postural control and balance [7]. Postural control and balance are functions of interactions between neural and musculoskeletal systems. Neural system elements involved in postural control include motion processes, like neuromuscular synergies, and sensory processes, such as vestibular and vision systems, and proprioception. In other words, postural control is achieved through a close and complex interaction between sensory inputs and appropriate motion responses, including motion system control and efficient muscular strength [8]. Studies on the elderly suggested that decreased flexibility and muscular strength lead to imbalance and increase the risk of falling, consequently resulting in reduced quality of life among this population [9].

Muscle weakness is a prevalent condition among the elderly [10]. Training improves the power of lower extremities and increases stable balance. Physical activities maintain muscle performance and mass at a high level and decrease the effects of age-related transformations in the elderly [11]. Studies indicated a direct relationship between falling and a low range of motion in joints. Decreased range of motion results from the rigidity of muscle-tendon unit and tissues around the joints, particularly in the lower extremities, which affect the dynamics of this muscle in walking and increases the risk of falling [12]. Studies on the relationship between muscle strength and range of motion, and balance and risk of falling are abundant. 
Jung et al. examined the relationship between muscle strength and range of motion, and the level of physical activity in the elderly with a history of falling [13]. They suggested a significant decrease in the range of motion hip extension, ankle dorsiflexion, and ankle plantar flexion. The scholars also reported a significant decrease in the muscle strength of knee flexion and extension in the subjects. Granacher et al. investigated the role of core stability strength in preventing falls in the elderly and reported favorable results in this respect [14]. Chiacchiero et al. examined the relationship between an inactive range of motion, flexibility, and balance in the elderly with and without a history of falling [15]; they revealed a significant decrease in the range of motion, hip extension, hip internal rotation, hip abduction, ankle dorsiflexion, and the length of twin muscles in the elderly with a history of falling.

Aging is essential in industrial and developing communities, like Iran. Moreover, the effects of aging are significant in the life quality of the elderly. Thus, it is essential to develop schemes to identify determinants of motion factors and changes in their daily living activities. A large body of literature explored the role of muscle strength and range of motion of some muscles and joints in the lower extremities in maintaining balance among the elderly. However, studied overlooked the effects of all muscles and joints to understand which ones are the least or the most effective on maintaining balance and falling risk in the elderly. Therefore, it is essential to determine the effects of every single muscle and joint in the lower extremities.

Such data could be beneficial to design strength and flexibility training protocols for lower extremities, particularly the most effective muscles, and joints, to improve balance and decrease the risk of falling in the elderly. Furthermore, studied explored the relationship between muscle strength and the range of motion in certain muscles in the lower extremities, and the level of physical activities, or balance, or certain falling factors, and compared the elderly with and without a history of falling. However, the present study used static and dynamic balance tests to examine the relationship between muscle strength and the range of motion in all muscles and joints in the lower extremities, with balance and the risk of falling in the elderly.

\section{Materials and Methods}

This was a correlative cross-sectional study that included 65 healthy male subjects (Mean \pm SD age: $65.64 \pm 4.95$ y) from Rasht City, Iran, who were selected by convenience sampling technique. The study subjects were socially active and independently performed their daily living activities. Those with a history of illness or motion problems were identified by a questionnaire and excluded from the study.

The inclusion criteria of the study were the age of $\geq 60$ years, independence in performing daily living activities, the ability to walk a route of $12 \mathrm{~m}$ with/without help, the ability to follow and respond to instructions without help, willingness to participate in the study, no use of neurologic drugs that affect balance, no history of lower extremities injuries within the last 6 months, no muscular or neurological problems, no visual impairment, no postural disorders that disrupt the study, no history of surgery within the previous year, and no fracture occurrence in the lower or upper extremities a year before the survey.

The study was approved by the Ethics Committee of the Faculty of Physical Education, Guilan University. The study participants were informed of the purpose, procedure, and measures of the study and voluntarily entered the study with written consent. No harm threatened the study participants. Sharpened Romberg test (reliability for eyes open: 0.91-0.90; for eyes closed: 0.76-0.77) was used to measure static balance, while the Timed Get Up and Go test was used to measure dynamic balance (reliability: 0.99) [4].

Berg Balance Scale (BBS) was used to measure the risk of falling. It is a 14-item scale that measures balance in older adults and includes simple movements (sitting to standing, standing unsupported, transfers) to complex movements (standing with feet together, turning 360 degrees, \& standing on one foot). Each item consists of a five-point scale, ranging 0-4. The total score of 56 indicates excellent balance. The internal and external validity of the test was obtained as 0.98 and 0.99 , respectively [9].

The isometric strength of muscles was measured using a manual dynamometer and the Mentiplay method [16]. In all tests, a stabilizing strap attached to a fixed shaft was used to fix the muscle and eliminate the effects of the subject's strength. The dynamometer was placed under the strap, and its power was calculated. Each test was repeated three times, and the study subjects were requested to use all their strengths in performing the task. Contractions lasted about 5 seconds, each with $15 \mathrm{sec}$ onds of rest. The score was calculated as follows:

$$
\text { Muscle strength }=\frac{\text { The amount of weight } \times 9.8}{\text { Subject's weight }} \times 100
$$


Eight muscle groups were selected for measuring muscle strength, as follows: hip extensor, hip abduction, hip flexor, hip adductor, hamstring, quadriceps, ankle dorsiflexion, and ankle plantar flexion. The motion range was measured using a $180^{\circ}$ Universal goniometer proposed by Clarkson [17]. The range of motion of the lower extremities was measured in the hip extension, hip abduction, hip flexion, hip adduction, knee flexion, ankle dorsiflexion, and ankle plantar flexion.

The Shapiro-Wilk test was used to measure the normal distribution of data. All the data were normally distributed. Pearson's correlation coefficient was used to examine the relationship between muscle strength and the range of motion of the lower extremities, and balance and the risk of falling. Multivariate regression analysis was used to determine the regression line. The obtained data were analyzed by SPSS.

\section{Results}

Table 1 presents the Mean \pm SD scores of the descriptive information of study subjects. Table 2 lists the correlation of muscle strength of lower extremities with static and dynamic balance and the risk of falling. These results demonstrated that the muscle strength of hip extension, hip abduction, hamstring, quadriceps, and ankle plantarflexion had a significant relationship with static balance while being negatively related to dynamic balance $(\mathrm{P} \leq 0.05)$. In other words, increased muscle strength improves static balance while decreasing dynamic balance and the risk of falling. There was also a significant and negative relationship between the muscle strength of ankle dorsiflexion and dynamic balance and the risk of falling $(\mathrm{P} \leq 0.05)$. Thus, increased muscle strength decreased the risk of falling and improved dynamic balance (Table 2)

Table 1. Demographic characteristics of the study participants

\begin{tabular}{cc}
\hline Variables & Mean \pm SD \\
\hline Height $(\mathrm{cm})$ & $165.10 \pm 9.43$ \\
Weight $(\mathrm{kg})$ & $72.08 \pm 12.63$ \\
Age $(\mathrm{y})$ & $68.64 \pm 4.95$ \\
$*$ BMI $\left(\mathrm{kg} / \mathrm{m}^{2}\right)$ & $25.31 \pm 4.31$ \\
\hline
\end{tabular}

${ }^{\star}$ BMI: Body Mass Index.

PHYSICAL TREATMENTS

Table 2. Pearson correlation coefficient in the relationship between muscle strength of lower extremities with static and dynamic balance and risk of falling in Elderly.

\begin{tabular}{|c|c|c|c|c|c|c|c|c|c|}
\hline \multicolumn{10}{|c|}{ Muscle Strength $\left(\mathrm{N} / \mathrm{m}^{2}\right)$} \\
\hline \multirow{3}{*}{ Variables } & \multirow{3}{*}{ Mean $\pm S D$} & \multicolumn{4}{|c|}{ Static Balance (Time) } & \multirow{2}{*}{\multicolumn{2}{|c|}{ Dynamic Balance (Time) }} & \multirow{2}{*}{\multicolumn{2}{|c|}{ Risk of Falling }} \\
\hline & & \multicolumn{2}{|c|}{ Eyes Open } & \multicolumn{2}{|c|}{ Eyes Closed } & & & & \\
\hline & & $r$ & $r^{2}$ & $r$ & $r^{2}$ & $r$ & $r^{2}$ & $\mathbf{r}$ & $r^{2}$ \\
\hline Hip abduction & $62.40 \pm 8.22$ & $0.40 *$ & 0.16 & $0.45^{*}$ & 0.20 & $-0.31 *$ & 0.09 & $-0.58^{*}$ & 0.33 \\
\hline Hip adduction & $56.54 \pm 7.49$ & 0.19 & 0.03 & 0.11 & 0.01 & -0.16 & 0.02 & -0.3 & 0.09 \\
\hline Hip Flection & $71.89 \pm 7.57$ & 0.23 & 0.05 & 0.21 & 0.04 & -0.23 & 0.05 & -0.14 & 0.01 \\
\hline Hip extension & $59.82 \pm 7.64$ & 0.65 & 0.42 & 0.67 & 0.44 & $-0.74^{*}$ & 0.54 & $-0.82^{*}$ & 0.67 \\
\hline Hamstring & $73.49 \pm 12.37$ & 0.52 & 0.27 & 0.55 & 0.30 & $-0.63 *$ & 0.39 & $-0.65^{*}$ & 0.42 \\
\hline Quadriceps & $135.89 \pm 24.13$ & 0.61 & 0.37 & 0.63 & 0.39 & $-0.46^{*}$ & 0.5 & $-0.68 *$ & 0.04 \\
\hline Plantar flexion & $49.00 \pm 7.55$ & 0.61 & 0.37 & 0.67 & 0.44 & $-0.74 *$ & 0.54 & $-0.65^{*}$ & 0.42 \\
\hline Dorsiflexion & $40.13 \pm 7.45$ & 0.18 & 0.03 & 0.12 & 0.01 & $-0.43^{*}$ & 0.18 & $-0.39 *$ & 0.15 \\
\hline
\end{tabular}

${ }^{*}$ Significant at $\mathrm{P} \leq 0.05$. 
Table 3. Pearson correlation coefficient in the relationship between range of motion in lower extremities with static and dynamic balance and risk of falling in Elderly

\begin{tabular}{|c|c|c|c|c|c|c|c|c|c|}
\hline \multicolumn{10}{|c|}{ Range of Motion (Degrees) } \\
\hline \multirow{3}{*}{ Variables } & \multirow{3}{*}{ Mean $\pm S D$} & \multicolumn{4}{|c|}{ Static Balance (Time) } & \multirow{2}{*}{\multicolumn{2}{|c|}{ Dynamic Balance (Time) }} & \multirow{2}{*}{\multicolumn{2}{|c|}{ Risk of Falling }} \\
\hline & & \multicolumn{2}{|c|}{ Eyes Open } & \multicolumn{2}{|c|}{ Eyes Closed } & & & & \\
\hline & & $r$ & $r^{2}$ & $r$ & $r^{2}$ & $r$ & $r^{2}$ & $r$ & $r^{2}$ \\
\hline Hip abduction & $23.85 \pm 2.14$ & 0.19 & 0.03 & 0.14 & 0.01 & -0.13 & 0.01 & -0.11 & 0.01 \\
\hline Hip adduction & $11.29 \pm 1.75$ & 0.21 & 0.04 & 0.12 & 0.01 & -0.13 & 0.01 & -0.14 & 0.02 \\
\hline Hip flection & $90.4 \pm 3.33$ & 0.24 & 0.05 & 0.16 & 0.02 & -0.23 & 0.05 & -0.21 & 0.04 \\
\hline Hip extension & $8.62 \pm 1.04$ & $0.53^{*}$ & 0.28 & $0.62 *$ & 0.38 & $-0.73^{*}$ & 0.53 & -0.63 & 0.39 \\
\hline Knee flection & $111.68 \pm 8.62$ & $0.51^{*}$ & 0.26 & $0.62^{*}$ & 0.38 & $-0.55^{*}$ & 0.3 & -0.69 & 0.47 \\
\hline Plantar flexion & $32.44 \pm 2.73$ & $0.76^{*}$ & 0.57 & $0.86^{*}$ & 0.73 & $-0.74^{*}$ & 0.54 & -0.83 & 0.68 \\
\hline Dorsiflexion & $13.88 \pm 2.13$ & $0.66^{*}$ & 0.43 & $0.61^{*}$ & 0.37 & $-0.51^{*}$ & 0.26 & -0.67 & 0.44 \\
\hline
\end{tabular}

${ }^{*}$ Significant at $\mathrm{P} \leq 0.05$.

PHYSICAL TREA $\ M E N T S$

Table 4. Regression equation results for predicting static and dynamic balance and the risk of falling in terms of muscle strength of lower extremities in the studied subjects

\begin{tabular}{cc}
\hline Variables & Model \\
\hline Static balance (eyes open) & $y=(0.242 \times 1)+(2.085 \times 3)+(0.212 \times 5)$ \\
Static balance (eyes closed) & $y=(0.055 \times 1)+(0.184 \times 2)+(0.142 \times 3)+(0.032 \times 5)$ \\
Dynamic balance & $y=(-1.027 \times 1)+(-0.517 \times 6)$ \\
Risk of falling & $y=(-2.047 \times 2)+(-0.041 \times 2)+(-0.241 \times 2)+(-1.177 \times 2)+(-0.032 \times 2)$ \\
\hline & PHYSICAL TREA $\mathbf{M E N T S}$
\end{tabular}

In the regression equations mentioned, y denotes static, dynamic balance and risk of falling and $x 1$ : hip abduction, $x 2$ : hip extension, x3: hamstring, x4: quadriceps, x5: plantar flexion, x6: dorsiflexion.

Table 5. Regression equation data for predicting static and dynamic balance and the risk of falling in terms of the range of motion of the lower extremities in the samples

\begin{tabular}{cc}
\hline Variables & Model \\
\hline Static balance (eyes open) & $y=(0.215 \times 1)+(2.122 \times 2)+(0.108 \times 3)$ \\
Static balance (eyes closed) & $y+0.184 \times 3$ \\
Dynamic balance & $y=(-0.37 \times 1)+(-1.13 \times 2)+(-0.071 \times 3)+(-0.432 \times 3)$ \\
Risk of falling & $y=(0.022 \times 1)+(-0.042 \times 3)$ \\
\hline & PHYSICAL TREA $\mathbf{M E N T S}$
\end{tabular}

In the regression equations mentioned, y denotes static, dynamic balance and risk of falling, and $x 1$ : hip extension, x2: knee flexion, x3: plantar ankle flexion, $x 4$ : ankle dorsiflexion.

Table 3 demonstrates the correlation between the range of motion joints of lower extremities with static and dynamic balance and the risk of falling. The relevant results suggested a positive and significant rela- tionship between the range of motion of hip extension, knee flexion, plantar flexion, and ankle dorsiflexion with static balance; while revealing a negative and significant relationship between these variables with dy- 
namic balance and the risk of falling $(\mathrm{P} \leq 0.05)$. In other words, the increased range of motion enhanced static balance, while decreasing the duration of dynamic balance and the risk of falling (Table 3).

Table 4 presents the regression line between the muscle strength of hip extension, hip abduction, hamstring, quadriceps, ankle dorsiflexion, and plantar flexion, and static and dynamic balance, and the risk of falling. The results of testing the hypothesis of independence of errors using the Durbin-Watson statistic indicated they were confirmed at 1.40 and 2.5. The achieved statistics suggested that the proposed model was significant for all the variables at $\mathrm{P} \leq 0.05$, and the model was linear. The value of tolerance in all states was $>0.05$, and no relation of linearity was observed in the study (Table 4).

Table 5 indicated the regression line between the range of motion of hip extension, knee flexion, ankle dorsiflexion, and plantar flexion, and static and dynamic balance, and the risk of falling. The results of testing the hypothesis of independence of errors, using DurbinWatson statistics, revealed that they were confirmed at 1.40 and 2.5. The obtained statistics demonstrated that the proposed model was significant for all the variables at $P \leq 0.05$, and the model was linear. The value of tolerance in all states was $>0.05$, and no relation of linearity was observed in the study (Table 5).

\section{Discussion}

The present study examined the relationship between muscle strength and the range of motion of the lower extremities, and balance and the risk of falling in the elderly. The obtained results indicated that the muscle strength of hip extension, hip abduction, hamstring, quadriceps, and ankle plantar flexion and dorsiflexion were significantly correlated with balance and decreased risk of falling in the elderly. This finding was consistent with those of Ding and Yang; they studied the effects of fatigue and muscle weakness of lower extremities on the risk of falling in older adults.

The authors created maximum isometric strength in the lower extremities of older adults and used a dynamometer to measure muscle strength in hamstring and quadriceps. Then, they used a particular treadmill to induce fatigue and muscle weakness. The study subjects walked and then ran slowly for 5 minutes on the treadmill until they felt tired. At that moment, their muscle strength of hamstring and quadriceps was measured by a dynamometer, indicating a significant decrease [18].
However, our results were in disagreement with those of Keskin et al. [19]; they examined risk factors associated with falling in the elderly with and without a history of falling. They reported no significant difference in the muscle strength of the knee extensors and flexors in the elderly with and without a history of falling. The present study results were in line with those of Jung et al. [13]; they reported that the muscle strength of the knee extensors and flexors significantly decreased in the elderly with a history of falls.

Menz at al. and Bok et al. also identified the decreased muscle strength of ankle plantar flexion as a risk factor for falling in the elderly $[20,21]$. Thus, the muscle strength of hip extension, hip abduction, hamstring, quadriceps, ankle dorsiflexion, and ankle plantarflexion affect balance and falling in older adults. Moreover, our results implied a significant relationship between the range of motion of hip flexion, knee flexion, plantar flexion, and ankle dorsiflexion, and balance and the risk of falling in the elderly.

This result was consistent with those of Jung et al., who reported a significant decrease in the range of motion, hip flexion, plantar flexion, and ankle dorsiflexion in the elderly who had fallen before [13]. Chiacchiero et al. revealed a significant decrease in the range of motion, hip extension, hip internal rotation, hip abduction, ankle dorsiflexion, and the length of twin muscles in the elderly with a history of falling [15]. Delbaere et al. used the hypothesis of a vicious circle to support these findings [22]. They explained that the fear of falling makes people less willing to engage in routine activities, which in turn, increased muscle strength and the range of motion and finally decreased balance and increased the risk of falling.

Mecagni et al. examined the relationship between age and balance, and the range of motion in 64-87-year-olds and stated that the motion range of ankle directly impacts balance in the elderly [23]. They also reported that increased age decreases balance and the range of motion. Kemoun et al. reported that a decreased range of motion in the ankle dorsiflexion significantly decreases balance and increases the odds of falling in the elderly [24]. Therefore, the range of motion, hip extension, knee flexion, plantar flexion, and ankle dorsiflexion affect balance and decrese the risk of falling in the elderly.

Limitations of the present study included inaccessibility of samples, subject restrictions, controlling the environment temperature, psychological factors, and encouraging subjects to participate in the study, limiting samples to male elderly, and the lack of control over so- 
cioeconomic, cultural, health, and dietary factors of the study subjects. Thus, it is suggested that similar studies be conducted on female subjects to regard the effects of gender. Those who have fallen could also be compared with those without a history of falls to understand how falling affects this relationship.

\section{Conclusion}

The obtained data suggested that the muscle strength of hip abduction, hip extension, hamstring, quadriceps, plantar flexion, ankle dorsiflexion and the range of motion, hip extension, knee flexion, plantar flexor, and ankle dorsiflexor have significant correlations with balance and the risk of falling in the elderly. Thus, researchers could develop intervening and training exercises to promote balance and decrease the risk of falls in the elderly. Such measures could help to improve their life quality and biopsychological well-being and encourage an active lifestyle among them.

\section{Ethical Considerations}

\section{Compliance with ethical guidelines}

This study was approved by the Ethics Committee of Guilan University.

\section{Funding}

This research received no specific grant from funding agencies in the public, commercial, or not-for-profit sectors.

\section{Authors' contributions}

All authors made substantial contributions to conception, design, acquisition, analysis, and interpretation of data.

\section{Conflict of interest}

The authors declared no conflicts of interest.

\section{Acknowledgments}

The authors highly appreciate all those who contributed to this research.

\section{References}

[1] Kinsella KG, Phillips DR. Global aging: The challenge of success. Washington DC: Population Reference Bureau; 2005.
[2] Gillespie LD, Robertson MC, Gillespie WJ, Sherrington C, Gates S, Clemson LM, et al. Interventions for preventing falls in older people living in the community. Cochrane Database of Systematic Reviews. 2012; (9):CD007146 [DOI:10.1002/14651858.CD007146.pub3]

[3] Ballesteros MF, Webb K, McClure RJ. A review of CDC's Web-based Injury Statistics Query and Reporting System (WISQARSTM): Planning for the future of injury surveillance. J Safety Res. 2017; 61:211-5. [DOI:10.1016/j. jsr.2017.01.001] [PMID] [PMCID]

[4] Lopes K, Costa D, Santos L, Castro D, Bastone A. Prevalence of fear of falling among a population of older adults and its correlation with mobility, dynamic balance, risk and history of falls. Brazilian Journal of Physical Therapy. 2009; 13(3):223-9. [DOI:10.1590/S1413-35552009005000026]

[5] Schmid AA, Acuff M, Doster K, Gwaltney-Duiser A, Whitaker A, Damush T, et al. Poststroke fear of falling in the hospital setting. Topics in Stroke Rehabilitation. 2009; 16(5):35766. [DOI:10.1310/tsr1605-357] [PMID]

[6] Berg K. Balance and its measure in the elderly: A review. Physiotherapy Canada. 1989; 41(5):240-6. [DOI:10.3138/ ptc.41.5.240]

[7] Nyman S, Ingram W, Sanders J, Thomas P, Thomas S, Vassallo M, et al. Randomised controlled trial of the effect of Tai Chi on postural balance of people with dementia. Clinical Interventions in Aging. 2019; 14:2017-29. [DOI:10.2147/CIA. S228931] [PMID] [PMCID]

[8] Kuo FC, Chang ST, Liao YY, Lee CH. Center of pressure trace and sensory components of the limits of stability test in older adults with vertebral compression fractures. Journal of Geriatric Physical Therapy. 2020; 43(1):24-31.

[9] Emilio EJ, Hita-Contreras F, Jiménez-Lara PM, LatorreRomán P, Martínez-Amat A. The association of flexibility, balance, and lumbar strength with balance ability: Risk of falls in older adults. Journal of Sports Science \& Medicine. 2014; 13(2):349-57.

[10] Rodacki AL, Souza RM, Ugrinowitsch C, Cristopoliski F, Fowler NE. Transient effects of stretching exercises on gait parameters of elderly women. Manual Therapy. 2009; 14(2):167-72. [DOI:10.1016/j.math.2008.01.006] [PMID]

[11] Kerrigan DC, Lee LW, Collins JJ, Riley PO, Lipsitz LA. Reduced hip extension during walking: Healthy elderly and fallers versus young adults. Archives of Physical Medicine and Rehabilitation. 2001; 82(1):26-30. [DOI:10.1053/ apmr.2001.18584] [PMID]

[12] Latham N, Liu Cj. Strength training in older adults: The benefits for osteoarthritis. Clinics in Geriatric Medicine. 2010; 26(3):445-59. [DOI:10.1016/j.cger.2010.03.006] [PMID] [PMCID]

[13] Jung H, Yamasaki M. Association of lower extremity range of motion and muscle strength with physical performance of community-dwelling older women. Journal of Physiological Anthropology. 2016; 35(1):30. [DOI:10.1186/s40101-0160120-8] [PMID] [PMCID]

[14] Granacher U, Gollhofer A, Hortobagyi T, Kressig RW, Muehlbauer T. The importance of trunk muscle strength for balance, functional performance, and fall prevention in seniors: A systematic review. Sports Medicine. 2013; 43(7):627 41. [DOI:10.1007/s40279-013-0041-1] [PMID] 
[15] Chiacchiero M, Dresely B, Silva U, DeLosReyes R, Vorik $B$. The relationship between range of movement, flexibility, and balance in the elderly. Topics in Geriatric Rehabilitation. 2010; 26(2):148-55. [DOI:10.1097/TGR.0b013e3181e854bc]

[16] Mentiplay BF, Tan D, Williams G, Adair B, Pua YH, Bower $\mathrm{KJ}$, et al. Assessment of isometric muscle strength and rate of torque development with hand-held dynamometry: Test-retest reliability and relationship with gait velocity after stroke. Journal of Biomechanics. 2018; 75:171-5. [DOI:10.1016/j.jbiomech.2018.04.032] [PMID]

[17] Clarkson HM. Joint motion and function assessment: A research-based practical guide. Philadelphia: Lippincott Williams \& Wilkins; 2005.

[18] Ding L, Yang F. Muscle weakness is related to slip-initiated falls among community-dwelling older adults. Journal of Biomechanics. 2016; 49(2):238-43. [DOI:10.1016/j.jbiomech.2015.12.009] [PMID]

[19] Keskin D, Borman P, Ersöz M, Kurtaran A, Bodur H, Akyüz M. The risk factors related to falling in elderly females. Geriatric Nursing. 2008; 29(1):58-63. [DOI:10.1016/j. gerinurse.2007.06.001] [PMID]

[20] Menz HB, Morris ME, Lord SR. Foot and ankle risk factors for falls in older people: A prospective study. The Journals of Gerontology Series A: Biological Sciences and Medical Sciences. 2006; 61(8):866-70. [DOI:10.1093/gerona/61.8.866] [PMID]

[21] Bok S-K, Lee TH, Lee SS. The effects of changes of ankle strength and range of motion according to aging on balance. Annals of Rehabilitation Medicine. 2013; 37(1):10-6 [DOI:10.5535/arm.2013.37.1.10] [PMID] [PMCID]

[22] Delbaere K, Crombez G, Vanderstraeten G, Willems T, Cambier D. Fear-related avoidance of activities, falls and physical frailty. A prospective community-based cohort study. Age and Ageing. 2004; 33(4):368-73. [DOI:10.1093/ ageing/afh106] [PMID]

[23] Mecagni C, Smith JP, Roberts KE, O'Sullivan SB. Balance and ankle range of motion in community-dwelling women aged 64 to 87 years: A correlational study. Physical Therapy. 2000; 80(10):1004-11. [DOI:10.1093/ptj/80.10.1004] [PMID]

[24] Kemoun G, Thoumie P, Boisson D, Guieu JD. Ankle dorsiflexion delay can predict falls in the elderly. Journal of Rehabilitation Medicine. 2002; 34(6):278-83. [DOI:10.1080/165019 702760390374] [PMID] 